\title{
Characteristics of the Distribution of Ferritin in Epithelial Ovarian Tumor Patients: Results of a Retrospective, Observational Study
}

\author{
Jinpeng Jiang1*, Sufei Wang1*, Lei Zhang1, Jinzhi Lu', Cunjian Yi1" \\ ${ }^{1}$ Department of Obstetrics and Gynecology, The First Affiliated Hospital of Yangtze University, Jingzhou, China \\ ${ }^{2}$ Department of Laboratory Medicine, The First Affiliated Hospital of Yangtze University, Jingzhou, China \\ Email: *1170445075@qq.com, ${ }^{*}$ cunjiany@163.com, ${ }^{*}$ Jinzhilu2015@163.com
}

How to cite this paper: Jiang, J.P., Wang, S.F., Zhang, L., Lu, J.Z. and Yi, C.J. (2018) Characteristics of the Distribution of Ferritin in Epithelial Ovarian Tumor Patients: Results of a Retrospective, Observational Study. Yangtze Medicine, 2, 51-61. https://doi.org/10.4236/ym.2018.22006

Received: February 26, 2018

Accepted: June 8, 2018

Published: June 11, 2018

Copyright (๑) 2018 by authors and Scientific Research Publishing Inc. This work is licensed under the Creative Commons Attribution International License (CC BY 4.0).

http://creativecommons.org/licenses/by/4.0/ (c) (i) Open Access

\begin{abstract}
Purpose: To investigate the ferritin distribution in epithelial ovarian cancer patients according to the FIGO stage in the prognosis of epithelial ovarian cancer. Method: All ovarian cancer patients were divided into two groups according their FIGO stage. Benign ovarian tumor patients were analyzed as the control. Serum ferritin, serum iron, and other related medical index were detected by automatic instruments for all patients. In addition, ferritin heavy chain (FHC) and ferritin light chain (FLC) proteins were detected by immunohistochemical staining in 60 epithelial ovarian cancer (EOC) patients and 30 benign ovarian tumor (BOT) patients, which were diagnosed in our department between 2011 and 2016. Results: The serum ferritin concentration was significantly higher in the EOC group than in the BOT group (172.56 \pm $99.39 \mathrm{ng} / \mathrm{mL}$ vs $78.18 \pm 43.06 \mathrm{ng} / \mathrm{mL} ; p<0.001)$. The average concentration of serum iron was slightly lower in the EOC group than in the BOT group $(11.11 \pm$ $5.68 \mu \mathrm{mol} / \mathrm{L}$ vs $14.92 \pm 6.36 \mu \mathrm{mol} / \mathrm{L} ; p<0.01)$. In addition, FHC and FLC proteins were highly expressed in EOC patients and localized at least in the cytoplasm and nucleus. Quantitative data of the FHC or FLC expression showed a significant increase in the average optical density in the benign ovarian tumor group compared with the FIGO I-II stage ovarian cancer group ( $p<$ 0.01 and $p<0.01$, respectively) as well as in the FIGO I-II stage ovarian cancer group compared with the FIGO III-IV stage ovarian cancer group $(p<0.05$ and $p<0.05$, respectively). In addition, FHC and FLC positive rates of $81.7 \%$ and $76.7 \%$, respectively, were found in the ovarian cancer group; the FLC positive rate in the FIGO I-II stage ovarian cancer group was lower than in the FIGO III-IV stage ovarian cancer group $(58.3 \%$ vs $86.1 \%$; $p<0.05)$; and the FHC positive rate in the FIGO I-II stage ovarian cancer group was lower than in the FIGO III-IV stage ovarian cancer group (70.8\% vs $88.9 \%)$, but this
\end{abstract}

\footnotetext{
*Authors contributed equally.

\#Corresponding authors.
} 
difference was not statistically significant $(p>0.05)$. Conclusion: Patients showed an overexpression of ferritin and a downregulation of serum iron correlated with the prognosis of epithelial ovarian cancer, which may be a new target for diagnosis and treatment of epithelial ovarian cancer.

\section{Keywords}

Ovarian Cancer, Ferritin, Ferritin Heavy Chain, Ferritin Light Chain, Serum Iron

\section{Introduction}

Ferritin is a multimeric globular protein of approximately $450 \mathrm{kDa}$ constituted by 24 subunits of the proteins ferritin heavy chain (FHC) and ferritin light chain (FLC), assembled in a nanocage with a central cavity where up to 4500 iron atoms can be stored [1]. Under normal conditions, ferritin can store intracellular iron in a non-toxic and readily available form, and it often plays an important role as major antioxidant molecule and has been long recognized to be essential in maintaining the cellular redox status [2] [3]. Recent studies have shown that a high expression of ferritin plays an important role in tumor invasion, tumor growth, and chemotherapeutic drug resistance [1] [4]. For example, Connor et al. (2015) pointed out that ferritin inhibits the death of breast cancer cells induced by oxidative stress [5]. In 2011, they found that ferritin (H chain) siRNA increases the chemotherapeutic effect of carmustine on the DNA damage of gliomas [6]. Faniello et al. (2011) found that cell proliferation, cell invasion, and protein expression were significantly reduced when the ferritin gene was silenced by proteomic analysis of MM07m melanoma cells. Observation of their tumor growth capacity in vivo, the injected with FHC-silenced MM07(m) cells mice showed a remarkable 4 -fold reduction compared to those who received the FHC-unsilenced MM07(m) counterpart [7].

Epithelial ovarian cancer is a serious gynecological with high mortality. More than $50 \%$ of the patients relapse and produce chemotherapeutic drug resistance, and the treatment of recurrent resistant ovarian cancer is rather difficult in clinic. It has been reported that patients with ovarian cancer exhibited iron deficiency, anemia, and an increased ferritin concentration [4] [8] [9] [10] [11]. In addition, studies have shown that ferritin is expressed extracellularly in serum plasma as well as intracellularly in the cytoplasm, nucleus, mitochondria, and other cell compartments [1] [2] [3] [9] [10] [11] [12]. In this study, we investigated the ferritin distribution in clinical epithelial ovarian tumor patients according to the FIGO stage, striving to provide more laboratory references for the diagnosis and treatment study of ovarian cancer.

\section{Material and Methods}

\subsection{Cases and Groups}

The present study is a retrospective, observational trial performed after approval 
by the ethics committee of The First Affiliated Hospital of Yangtze University. Ninety cases were selected from clinically diagnosed cases in The First Affiliated Hospital of Yangtze University between August 2011 and August 2016. The patients were divided into two groups: the first group contained 60 cases with epithelial ovarian cancer (46 serous adenocarcinoma and 14 mucinous adenocarcinoma) and the second group contained 30 cases of epithelial benign tumors as control (24 serous cystadenoma and 6 mucinous cystadenoma).

Inclusion criteria: 1) All cases had protopathic diseases and were pathologically examined to be epithelial ovarian cancer or epithelial ovarian benign tumors; 2) Patients were assessed at diagnosis before receiving any cancer treatment; 3) Operation notes were complete and sufficiently distinct to obtain the clinicopathological stage in accordance with the 2015 International Federation of Gynecology and Obstetrics (FIGO) standards of ovarian cancer. Available tissues of patients should be kept in our pathology department; 4) Essential information and laboratory test results were fully documented. Exclusion criteria: 1) Evidence of infections, chronic inflammatory disease, active bleeding, hemolysis, renal insufficiency, or hypothyroidism; 2) Known history of hematologic disorders, family history of thalassemia or hemocromatosis; 3) Treatment with Erythropoietin; iron or blood transfusion in the preceding 12 weeks; current iron, vitamin B12, or folate supplementation.

\subsection{Hematological Data Acquisition}

Parameters directly collected from previous medical records include red blood cell (RBC), hemoglobin (HB), mean corpuscular volume (MCV), mean corpuscular hemoglobin $(\mathrm{MCH})$, mean corpuscular hemoglobin concentration (MCHC), serum iron concentration, the liver enzymes alanine aminotransferase (ALT) and aspartate transaminase (AST), the kidney health indicator blood urea nitrogen (BUN), and albumin (ALB). Serum ferritin and cancer antigen 125 (CA125) were additionally examined using the saved blood samples collected at the time of the preoperative examinations and kept at $-80^{\circ} \mathrm{C}$ until sampling. Serum ferritin and CA125 were detected by the Cobas 6000 analyzer (Roche Diagnostics $\mathrm{GmbH}$, Germany) and the corresponding kit (Roche Diagnostics $\mathrm{GmbH}$, Germany).

\subsection{Immunohistochemical Staining}

Four- $\mu \mathrm{m}$ thick paraffin-embedded, formalin-fixed slides of de-identified human tissues were deparaffinized, and high-temperature heat pressure antigen retrieval $\left(120^{\circ} \mathrm{C}, 120 \mathrm{kPa}\right)$ was performed by tissue incubation in $0.01 \mathrm{~mol} / \mathrm{L}$ citrate buffer $(\mathrm{pH}=6.0)$ for $3 \mathrm{~min}$. The sections were then incubated with $5 \mu \mathrm{g} / \mathrm{mL}$ ferritin heavy chain (FHC) antibodies (ab65080, Abcam, Cambridge, United Kingdom) and $5 \mu \mathrm{g} / \mathrm{mL}$ ferritin light chain (FLC) antibodies (ab69090, Abcam, Cambridge, United Kingdom) at $4^{\circ} \mathrm{C}$ overnight and analyzed with an horseradish peroxidase-conjugated compact polymer system using $\mathrm{DAB}$ as the chromogen. The 
sections were then counterstained with hematoxylin and mounted with DPX.

\subsection{Image Analysis}

Imaging of immunohistochemically (IHC) stained tissue was performed using the imaging system HMIAS-2000W (Wuhan Champion Image Technology Co., Ltd., Wuhan, China) and the Olympus BX41 microscope (Olympus Corporation, Japan), and the positive rate and average optical density were calculated from the images.

Positive criteria: protein expression was assessed by scoring the percentage of the labelled cells. FHC and FLC expressions were quantified using a visual grading system based on the proportion of staining (percentage of positive tumor cells graded on a scale from 0 to $3: 0$, none; $1,1 \%-30 \% ; 2,31 \%-60 \% ; 3,>60 \%$ ) and the intensity of staining (graded on a scale of 0 to $3: 0$, none; 1 , weak staining; 2, moderate staining; 3 , strong staining). The product $\mathrm{PI}=\mathrm{P} \times \mathrm{I}$ of proportion (P) and intensity (I) of the staining ranged between 0 and 9 and was obtained as a combined parameter for each spot. For statistical analysis, a PI score of 0 - 3 was considered as negative expression and a PI score $>3$ was considered as positive expression [13]. Determination of the average optical density: three images of the oncocytic area with $100 \times$ magnification were randomly obtained from every IHC section to quantify the average optical density (AOD) using the Image-Pro Plus 6.0 software, and the AOD data were visualized in a box plot created by the Origin 8.5 software (OriginLab, Northampton, MA, USA).

\subsection{Statistical Analysis}

Continuous data are expressed as mean \pm standard deviation (S.D.), and differences between two groups were compared by Student's t-test. Differences in the positive rates were analyzed using chi-square tests. Continuity correction chi-square tests were performed for contingency values between 1 and 5, while Pearson's chi-square test was performed for contingency values larger than 5 . Values of $p<0.05$ were considered statistically significant. Computations were performed using SPSS version 19.0 (International Business Machines Corporation, New York City, United States of America).

\section{Results}

\subsection{Ovarian Tumor Patients Clinical Characteristics and Their Serum Ferritin Analysis}

All 90 ovarian tumor patients were treated in our hospital. Among these patients, 30 patients with an age range of 20 - 71 years (average $45.10 \pm 15.05$ ) had benign ovarian tumors (BOT), and 60 patients with an age range of 25 - 75 years (average $52.18 \pm 9.79$ ) had epithelial ovarian cancer (EOC). From the blood samples of ovarian cancer patients, ferritin blood levels and other clinical characteristics were analyzed in parallel. These other clinical characteristics involved the patients' age, red blood cell counts, the liver enzymes alanine aminotransfe- 
rase (ALT) and aspartate transaminase (AST), the kidney health indicator blood urea nitrogen (BUN), albumin (ALB), the ovarian cancer marker cancer antigen 125 (CA125), and the serum iron level.

Benign ovarian tumor patients and ovarian cancer patients exhibited differences in various parameters, except for blood urea nitrogen (BUN). For example, the red blood cell (RBC) counts among ovarian cancer groups were $3.93 \pm$ $0.47 \times 10^{12} / \mathrm{L}$, which was significantly smaller than the counts of $4.26 \pm 0.61 \times$ $10^{12} / \mathrm{L}$ in the benign ovarian tumor groups $(p<0.01$; Table 1$)$. The average concentration of hemoglobin (HB) was significantly lower in the EOC group than in the BOT group $(114.12 \pm 15.68 \mathrm{~g} / \mathrm{L}$ vs $122.68 \pm 15.13 \mathrm{~g} / \mathrm{L} ; p<0.05)$. In contrast, erythrocyte mean corpuscular volume (MCV), mean corpuscular hemoglobin $(\mathrm{MCH})$, and mean corpuscular hemoglobin concentration (MCHC) showed no significant differences between the two groups. The average concentrations of ALT and AST in the two groups were normal, while the EOC group showed a slightly higher value than the BOT group ( $p<0.05$ and $p<0.01$, respectively). The concentration of ALB was significantly lower in the EOC group than in the BOT group $(p<0.05)$. The average concentration of BUN was lower in the EOC group than in the BOT group, but this difference was not statistically significant $(p=0.074)$.

Evidently, the average concentration of the ovarian cancer marker CA125 was

Table 1. Ovarian tumor patients clinical characteristics.

\begin{tabular}{ccccc}
\hline Variable & $\begin{array}{c}\text { Benign Ovarian Tumor } \\
(\mathbf{n}=\mathbf{3 0})\end{array}$ & $\begin{array}{c}\text { Ovarian Cancer } \\
(\mathbf{n}=60)\end{array}$ & $\mathrm{T}$ & $\boldsymbol{p}$ \\
\hline Number & 30 & 60 & & \\
Age, years (range) & $45.10 \pm 15.05$ & $52.18 \pm 9.79$ & 2.342 & $0.024^{\star}$ \\
HB, g/L & $(20-71)$ & $(25-75)$ & & \\
RBC, $\times 10^{12} / \mathrm{L}$ & $122.68 \pm 15.13$ & $114.12 \pm 15.68$ & 2.470 & $0.015^{*}$ \\
MCV, fL & $4.26 \pm 0.61$ & $3.93 \pm 0.47$ & 2.779 & $0.007^{\star *}$ \\
MCH, Pg & $88.35 \pm 7.41$ & $88.49 \pm 4.93$ & 0.100 & 0.921 \\
MCHC, g/L & $29.03 \pm 2.80$ & $29.06 \pm 1.97$ & 0.052 & 0.958 \\
ALT, U/L & $328.47 \pm 10.70$ & $328.40 \pm 11.33$ & 0.027 & 0.979 \\
AST, U/L & $10.30 \pm 7.86$ & $14.50 \pm 9.19$ & 2.140 & $0.035^{*}$ \\
BUN, mmol/L & $18.13 \pm 4.17$ & $23.8 \pm 10.04$ & 2.961 & $0.004^{* *}$ \\
ALB, g/L & $5.01 \pm 1.78$ & $4.41 \pm 1.29$ & 1.806 & 0.074 \\
CA125, U/mL & $44.83 \pm 9.70$ & $41.80 \pm 4.38$ & 2.046 & $0.044^{*}$ \\
Serum ferritin, & $17.91 \pm 14.97$ & $255.64 \pm 246.45$ & 5.264 & $0.000^{* *}$ \\
ng/mL & $78.18 \pm 43.06$ & $172.56 \pm 99.39$ & 6.272 & $0.000^{* *}$ \\
Serum iron, $\mu \mathrm{mol} / \mathrm{L}$ & $14.92 \pm 6.36$ & $11.11 \pm 5.68$ & 2.883 & $0.005^{* *}$ \\
\hline
\end{tabular}

Results are considered significant for values of $p<0.05^{*}$ and highly significant for $p<0.01^{* *}$. Abbreviations: HB: hemoglobin; RBC: red blood cell; MCV: mean corpuscular volume; $\mathrm{MCH}$ : mean corpuscular hemoglobin; MCHC: mean corpuscular hemoglobin concentration; ALT: alanine aminotransferase; AST: aspartate transaminase; BUN: blood urea nitrogen; ALB: albumin; CA125: cancer antigen 125. 
much higher in the EOC group than in the BOT group $(255.64 \pm 246.45 \mathrm{U} / \mathrm{mL}$ vs $17.91 \pm 14.97 \mathrm{U} / \mathrm{mL} ; p<0.01)$. Similarly, the level of serum ferritin, one of the storage forms of iron, was significantly higher in the EOC group than in the BOT group $(172.56 \pm 99.39 \mathrm{ng} / \mathrm{mL}$ vs $78.18 \pm 43.06 \mathrm{ng} / \mathrm{mL} ; p<0.001)$. In contrast, the average concentration of serum iron was slightly lower in the EOC group than in the BOT group $(11.11 \pm 5.68 \mu \mathrm{mol} / \mathrm{L}$ vs $14.92 \pm 6.36 \mu \mathrm{mol} / \mathrm{L} ; p<$ 0.01 ), and this difference was statistically significant (Table 1 ).

\subsection{Evaluations of Secreted Serum Ferritin and Serum Iron in Ovarian Cancer Patients According to the FIGO Clinical Stage}

As mentioned above, the serum ferritin level was increased, and the serum iron level was decreased in the EOC group. To further ascertain the levels of secreted serum ferritin and serum iron in EOC patients with different FIGO clinical stages, the patients were divided according their FIGO stage into two groups with either FIGO stage I-II or III-IV (Table 2). The average concentration of serum ferritin calculated for the EOC group with FIGO stage III-IV was higher than that for the group with FIGO I-II stage $(188.76 \pm 102.11 \mathrm{ng} / \mathrm{mL}$ vs $148.27 \pm$ $91.92 \mathrm{ng} / \mathrm{mL}$ ). The average concentration of serum iron of the group with FIGO stage III-IV was lower than that of the group with FIGO stage I-II (10.25 \pm 5.66 $\mu \mathrm{mol} / \mathrm{L}$ vs $12.39 \pm 5.58 \mu \mathrm{mol} / \mathrm{L})$. Furthermore, the average concentration of $\mathrm{HB}$ for the FIGO III-IV stage group was lower than for the FIGO I-II stage group $(112.34 \pm 15.46 \mathrm{~g} / \mathrm{L}$ vs $116.80 \pm 15.96 \mathrm{~g} / \mathrm{L})$, and the average concentration of RBC in the FIGO III-IV stage group was slightly lower than in the FIGO I-II stage group $\left(3.89 \pm 0.49 \times 10^{12} / \mathrm{L}\right.$ vs $\left.4.00 \pm 0.45 \times 10^{12} / \mathrm{L}\right)$. All of these data show a difference between the FIGO III-IV and I-II stage groups of EOC patients, although the difference of none of the examined parameters was statistically significant $(p>0.05)$. In contrast, the average concentrations of $\mathrm{MCV}, \mathrm{MCH}$, and MCHC in the FIGO III-IV and I-II stage groups were nearly identical and did not show any significant differences (Table 2).

Table 2. Evaluations of serum ferritin and serum iron in ovarian cancer patients according to their FIGO clinical stage.

\begin{tabular}{ccccc}
\hline \multirow{2}{*}{ Variable } & \multicolumn{2}{c}{ FIGO stage } & \multirow{2}{*}{ T } & \multirow{p}{*}{$\boldsymbol{p}$} \\
\cline { 2 - 3 } & I-II $(\mathbf{n}=\mathbf{2 4})$ & III-IV $(\mathbf{n}=\mathbf{3 6})$ & & \\
\hline Serum ferritin, $\mathrm{ng} / \mathrm{mL}$ & $148.27 \pm 91.92$ & $188.76 \pm 102.11$ & 1.565 & 0.123 \\
Serum iron, $\mu \mathrm{mol} / \mathrm{L}$ & $12.39 \pm 5.58$ & $10.25 \pm 5.66$ & 1.438 & 0.156 \\
$\mathrm{HB}, \mathrm{g} / \mathrm{L}$ & $116.80 \pm 15.96$ & $112.34 \pm 15.46$ & 1.078 & 0.285 \\
$\mathrm{RBC}, \times 10^{12} / \mathrm{L}$ & $4.00 \pm 0.45$ & $3.89 \pm 0.49$ & 0.865 & 0.391 \\
MCV, f L & $88.20 \pm 2.94$ & $88.68 \pm 5.93$ & 0.368 & 0.714 \\
MCH, Pg & $29.05 \pm 1.60$ & $29.06 \pm 2.21$ & 0.021 & 0.983 \\
MCHC, g/L & $329.50 \pm 14.86$ & $327.67 \pm 8.35$ & 0.549 & 0.587 \\
\hline
\end{tabular}

Abbreviations: FIGO: International Federation of Gynecology and Obstetrics; HB: hemoglobin; RBC: red blood cell; MCV: mean corpuscular volume; $\mathrm{MCH}$ : mean corpuscular hemoglobin; MCHC: mean corpuscular hemoglobin concentration. 


\subsection{Expression of Ferritin and Its Subtype Positive Rate According to the Ovarian Cancer FIGO Clinical Stage}

To investigate the expression of ferritin and its subtype positive rate, the two ferritin subtypes ferritin heavy chain (FHC) and ferritin light chain (FLC) were detected by immunohistochemical methods in benign ovarian tumor and epithelial ovarian cancer patients. As shown in Figure 1, FHC and FLC were highly expressed in EOC patients and were localized in the cytoplasm and nucleus. The average optical density was obtained and analyzed, which revealed that the expressions of FHC and FLC in the EOC group was increased compared with the BOT group: the average optical density of FHC in the BOT group was $0.172 \pm$ 0.051 , whereas values of $0.241 \pm 0.077$ and $0.277 \pm 0.057$ were determined for the FIGO I-II and III-IV stage EOC groups, respectively, which presents a statistically significant gradual increase $(\mathrm{t}=3.815, p<0.01$ and $\mathrm{t}=1.944, p<0.05$, respectively). In addition, the average optical density of FLC in the BOT group was $0.163 \pm 0.042$, whereas values of $0.220 \pm 0.089$ and $0.265 \pm 0.063$ were determined for the FIGO I-II and III-IV stage EOC groups, respectively, which also presents a statistically significant gradual increase between these groups $(\mathrm{t}=$ 2.928, $p<0.01$ and $\mathrm{t}=2.117, p<0.05$, respectively; Figure 1).

Furthermore, the positive rates of FHC and FLC were calculated according to the ovarian cancer FIGO clinical stage. As shown in Table 3, the positive rates of FHC and FLC in the EOC group were $81.7 \%$ and $76.7 \%$, respectively. The positive rate of FHC in the FIGO III-IV stage EOC group was higher than that in the FIGO I-II stage group ( $88.9 \%$ vs $70.8 \%$ ), but this difference was not statistically significant. The positive rate of FLC in the FIGO III-IV stage EOC group was higher than that in the FIGO I-II stage EOC group ( $86.1 \%$ vs $58.3 \%)$, and this difference was statistically significant $(p<0.05$; Table 3$)$.

\section{Discussion}

Ferritin can be expressed in its intracellular iron cargo protein form or its extracellular form of secreted ferritin [1] [2] [3]. High expression of ferritin in cancer tissue plays an important role in tumor invasion, tumor growth, and chemotherapeutic drug resistance, and secreted ferritin could be detected in the serum and was considered as tumor marker [4]-[12]. However, some studies demonstrated the characteristics of the ferritin distribution in epithelial ovarian tumor patients. To the best of our knowledge, the present study is the first to show that patients show ferritin overexpression and serum ion downregulation as the tumor grows, which presents a potential new target for diagnosis and treatment of epithelial ovarian cancer.

The present study evaluates the prevalence of cancer-related anemia in a large cohort of oncology patients with cancers of the ovaries. For example, our data showed that both the red blood cell (RBC) count and the average concentration of hemoglobin (HB) were significantly decreased among ovarian cancer groups compared with benign ovarian tumor groups. $\mathrm{MCV}, \mathrm{MCH}$, and $\mathrm{MCHC}$ showed 
(a)


Epithelial Ovarian Cancer (I-II) Epithelial Ovarian Cancer (III-IV)
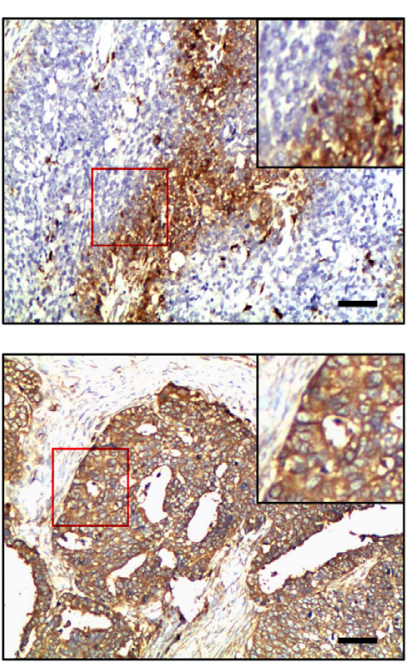


(b)
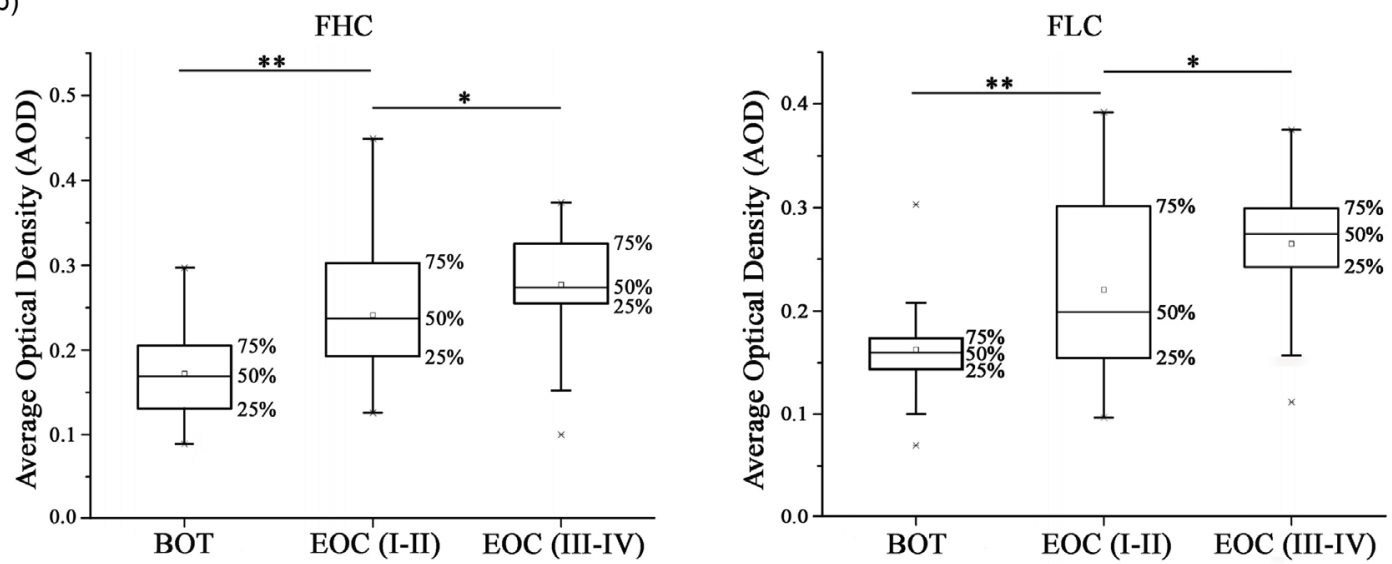

Figure 1. Ferritin heavy chain (FHC) and ferritin light chain (FLC) proteins were highly expressed in epithelial ovarian cancer patients and benign ovarian tumor patients. (a) Immunohistochemistry images of FHC and FLC expression (original magnification, $\times 100$ ). The inset shows the magnification of the red rectangular region. Black scale bars represent $200 \mu \mathrm{m}$. (b) Average optical density of panel A $\left({ }^{*} p<0.05,{ }^{* *} p<0.01\right)$.

no significant differences between the two groups. In addition, the average concentration of the liver enzymes ALT and AST in the two groups were normal while the EOC group showed a slightly higher concentration than the BOT group. In contrast, the concentration of albumin (ALB) was lower in the EOC group than in the BOT group. Anemia and nutrition deficiency in oncology patients is often considered a side effect of cancer therapy [8], and our study supported that anemia and nutrition deficiency may occur in ovarian cancer patients before any antineoplastic treatment.

We found that the serum iron concentration decreased with the tumor growth. We hypothesized that the tumor has an intrinsic need for iron, and some evidence supports this hypothesis. Some studies showed that cancer tissue usually has an increased iron uptake and a decreased iron release and usually exhibits an iron deficiency in the serum and an iron overload within the tumor 
Table 3. Ferritin subtype expression positive rate according to the ovarian cancer FIGO clinical stage.

\begin{tabular}{ccccccccc}
\hline \multirow{2}{*}{ Variable } & \multicolumn{2}{c}{ FHC } & & \multicolumn{2}{c}{ FLC } & & \\
\cline { 2 - 3 } & $\begin{array}{c}\text { Positive, } \\
\mathrm{n}(\%)\end{array}$ & $\begin{array}{c}\text { Negative, } \\
\mathrm{n}(\%)\end{array}$ & $x^{2}$ & $p$ & $\begin{array}{c}\text { Positive, } \\
\mathrm{n}(\%)\end{array}$ & $\begin{array}{c}\text { Negative, } \\
\mathrm{n}(\%)\end{array}$ & $x^{2}$ & $p$ \\
\hline Ovarian cancer & $49(81.7)$ & $11(18.3)$ & & & $46(76.7)$ & $14(23.3)$ & \\
Ovarian cancer (I-II) & $17(70.8)$ & $7(29.2)$ & 2.045 & 0.153 & $14(58.3)$ & $10(41.7)$ & 5.926 & 0.015 \\
Ovarian cancer (III-IV) & $32(88.9)$ & $4(11.1)$ & & & $31(86.1)$ & $5(13.9)$ & & \\
\hline
\end{tabular}

FIGO: International federation of gynecology and obstetrics. FHC: ferritin heavy chain; FLC: ferritin light chain.

[1] [2]. Cancer antigen 125 (CA125) is a traditional marker for the diagnosis of ovarian cancer. In the present study, a significant increase of CA125 was clearly detected in the EOC groups. At the same time, serum ferritin, one of the storage forms of iron, was analyzed and showed a similar increase in the EOC groups. Some studies have demonstrated an increase of serum ferritin in ovarian cancer patients [9] [10], which is consistent with our results. Furthermore, we calculated the concentration of serum ferritin for EOC patients with different FIGO clinical stages. Our results showed that the increase of serum ferritin, the extracellular form of secreted ferritin, is correlated with the prognosis of ovarian cancer. In addition, we analyzed the expression of ferritin in its intracellular form in epithelial ovarian tumor patients with immunohistochemical methods, revealing a higher ferritin expression in ovarian cancer patients compared with benign ovarian tumor patients. Analysis of the average optical density showed that the expression of ferritin heavy chain (FHC) and ferritin light chain (FLC) proteins in the EOC group gradually increased from BOT group, over FIGO I-II stage EOC patients, to FIGO III-IV stage EOC patients. These results showed that the expression of ferritin is positively correlated with tumor growth. As mentioned above, recent studies revealed that the higher ferritin expression in malignant tumors is related to myelodysplastic syndromes [14], cancer metastasis [15] [16], drug resistance [5] [6], and poor prognosis [17]. The higher ferritin expression determined in our study makes further investigations necessary to elucidate the function of ferritin in different compartments of cancer cells.

\section{Conclusion}

Overall, we conclude that an abnormal distribution of ferritin was found in epithelial ovarian cancer patients, and ferritin overexpression and serum iron downregulation in epithelial ovarian cancer were consistent with the cancer prognosis, indicating that ferritin may be a new target for diagnosis and treatment of epithelial ovarian cancer.

\section{Acknowledgements}

We acknowledge the excellent laboratory work performed by the technicians of the pathology department. This study was supported by the Iron-deficiency \& 
Iron-deficiency Anemia Research Grant project and Jingzhou science and technology development plan (key projects, 2015AC45 and 2016AE51-2), as well as the Hubei Province health and family planning scientific research project (key project, WJ2017Z024).

\section{References}

[1] Torti, S.V. and Torti, F.M. (2013) Iron and Cancer: More Ore to Be Mined. Nature Reviews Cancer, 13, 342-355. https://doi.org/10.1038/nrc3495

[2] Arosio, P. and Levi, S. (2002) Ferritin, Iron Homeostasis, and Oxidative Damage. Free Radical Biology \& Medicine, 33, 457-463. https://doi.org/10.1016/S0891-5849(02)00842-0

[3] Theil, E.C. (1987) Ferritin: Structure, Gene Regulation, and Cellular Function in Animals, Plants, and Microorganisms. Annual Review of Biochemistry, 56, 289-315. https://doi.org/10.1146/annurev.bi.56.070187.001445

[4] Basuli, D., Tesfay, L., Deng, Z., et al. (2017) Iron Addiction: A Novel Therapeutic Target in Ovarian Cancer. Oncogene, 36, 4089-4099. https://doi.org/10.1038/onc.2017.11

[5] Buranrat, B. and Connor, J.R. (2015) Cytoprotective Effects of Ferritin on Doxorubicin-Induced Breast Cancer Cell Death. Oncology Reports, 34, 2790-2796. https://doi.org/10.3892/or.2015.4250

[6] Liu, X., Madhankumar, A.B., Slagle-Webb, B., et al. (2011) Heavy Chain Ferritin siRNA Delivered by Cationic Liposomes Increases Sensitivity of Cancer Cells to Chemotherapeutic Agents. Cancer Research, 71, 2240-2249. https://doi.org/10.1158/0008-5472.CAN-10-1375

[7] Sanzo, M.D., Gaspari, M., Misaggi, R., et al. (2011) H Ferritin Gene Silencing in a Human Metastatic Melanoma Cell Line: A Proteomic Analysis. Journal of Proteome Research, 10, 5444-5453. https://doi.org/10.1021/pr200705z

[8] Macciò, A., Madeddu, C., Gramignano, G., et al. (2015) The Role of Inflammation, Iron, and Nutritional Status in Cancer-Related Anemia: Results of A Large Prospective Observational Study. Haematologica, 100, 124.

https://doi.org/10.3324/haematol.2014.112813

[9] Gulec, U.K., Paydas, S., Guzel, A.B., et al. (2012) Comparative Analysis of CA 125, Ferritin, Beta-2 Microglobulin, Lactic Dehydrogenase Levels in Serum and Peritoneal Fluid in Patients with Ovarian Neoplasia. Medical Oncology, 29, 2937-2943. https://doi.org/10.1007/s12032-012-0165-4

[10] Pinto, V., Marinaccio, M., Garofalo, S., et al. (1997) Preoperative Evaluation of Ferritinemia in Primary Epithelial Ovarian Cancer. Tumori, 83, 927-929.

https://doi.org/10.1177/030089169708300611

[11] Lobello, N., Biamonte, F., Pisanu, M.E., et al. (2016) Ferritin Heavy Chain Is a Negative Regulator of Ovarian Cancer Stem Cell Expansion and Epithelial to Mesenchymal Transition. Oncotarget, 7, 62019-62033. https://doi.org/10.18632/oncotarget.11495

[12] Bauckman, K., Haller, E., Taran, N., et al. (2015) Iron Alters Cell Survival in a Mitochondria-Dependent Pathway in ovarian Cancer Cells. Biochemical Journal, 466, 401-413. https://doi.org/10.1042/BJ20140878

[13] Maréchal, R., Demetter, P., Nagyet, N., et al. (2009) High Expression of CXCR4 May Predict Poor Survival in Resected Pancreatic Adenocarcinoma. British Journal of Cancer, 100, 1444-1451. https://doi.org/10.1038/sj.bjc.6605020 
[14] Pileggi, C., Di, S.M., Mascaro, V., et al. (2017) Role of Serum Ferritin Level on Overall Survival in Patients with Myelodysplastic Syndromes: Results of a Meta-Analysis of Observational Studies. Plos One, 12, e0179016. https://doi.org/10.1371/journal.pone.0179016

[15] Tripathi, P.K. and Chatterjee, S.K. (1996) Elevated Expression of Ferritin H-Chain mRNA in Metastatic Ovarian Tumor. Cancer Investigation, 14, 518-526. https://doi.org/10.3109/07357909609076897

[16] Zhang, K.H., Tian, H.Y., Xia, G., et al. (2009) Ferritin Heavy Chain Mediated Iron Homeostasis and Subsequent Increased Reactive Oxygen Species Production Are Essential for Epithelial-Mesenchymal Transition. Cancer Research, 69, 5340-5348. https://doi.org/10.1158/0008-5472.CAN-09-0112

[17] Lorenzi, M., Lorenzi, B. and Vernillo, R. (2006) Serum Ferritin in Colorectal Cancer Patients and Its Prognostic Evaluation. The International journal of Biological Markers, 21, 235-241. https://doi.org/10.1177/172460080602100407 